\title{
Knee Acoustic Emission Characteristics of the Healthy and the Patients with Osteoarthritis Using Piezoelectric Sensor
}

\author{
Dagyeong Choi, ${ }^{1}$ Soonjae Ahn, ${ }^{1}$ Jeseong Ryu, ${ }^{1}$ Mitsuo Nagao, ${ }^{2}$ and Youngho Kim ${ }^{1 *}$ \\ ${ }^{1}$ Department of Biomedical Engineering and Institute of Medical Engineering, Yonsei University, \\ 1 Yeonsedae-gil, Maeji-ri, Heungeop-myeon, Wonju-si, Gangwon-do 26493, Republic of Korea \\ ${ }^{2}$ Department of Mechanical Engineering, College of Engineering, Nihon University, \\ 1 Nakagawara, Rokusada, Tamura, Koriyama, Fukushima 963-8642, Japan
}

(Received May 10, 2017; accepted February 16, 2018)

Keywords: osteoarthritis, acoustic emission, piezoelectric sensor

Osteoarthritis (OA) is one of the most common causes of disability in elderly individuals. Although X-rays, MRIs, and arthroscopy are widely used to examine OA, they do not provide dynamic information on activity-based joint integrity. In this study, acoustic emission (AE) in healthy individuals and OA patients was determined using piezoelectric sensors. Twenty normal subjects and fourteen OA patients were asked to perform weight-bearing (stand-to-sit) and non-weight-bearing (extension to flexion) exercises for $4 \mathrm{~s}$ each. Sensors were attached to the medial and lateral epicondyles of the tibia and the front of the patella. An AE event was defined as the threshold crossing based on AE signals. The results indicated that $\mathrm{OA}$ and healthy groups exhibited signals at a frequency range corresponding to approximately $100 \mathrm{~Hz}-$ $10 \mathrm{kHz}$ and less than $1 \mathrm{kHz}$, respectively. The OA group exhibited an amplitude of AE signals and a number of AE events that exceeded those of the healthy group $(p<0.05)$. AE signals from lesions exhibited a higher $\mathrm{dB}$ value and a larger number of $\mathrm{AE}$ events than those at other locations. However, most patients exhibited significant increases in AE signal characteristics at the front of the patella. The results of this study can be helpful in the early diagnosis or easy monitoring of knee OA in daily lives.

\section{Introduction}

Osteoarthritis (OA) is a musculoskeletal disorder that is one of the most common causes of disabilities in elderly individuals. ${ }^{(1)}$ Changes in the musculoskeletal system due to aging are typically caused by muscle weakness, OA associated with decreased bone density, muscle atrophy caused by decreased muscle activity, and OA caused by degenerative changes in an articular cartilage. ${ }^{(2)} \mathrm{OA}$ corresponds to the inflammation of the joints in which joints are characterized by pathological reactions such as fever, pain, swelling, and redness. The principal role of synovial fluid surrounding the joints involves reducing friction between the articular cartilage structures of synovial joints during movement. The cartilage prevents damage of a part of the joint by concentrating the load at a specific point and also protects the tip of each bone like a sponge to act as a shock-buffering function. ${ }^{(3)}$ 
Synovial fluid in the joints contains nutrients that aid cartilage and gases such as carbon dioxide and oxygen. As a joint bends, the gas is pushed into the synovial fluid to form bubbles. At this point in time, the joint capsule suddenly becomes extremely narrow such that bubbles burst and the joint produces sound ${ }^{(4)}$ or this suddenly stretches ligaments, and the adhesive surface of the joints falls (although this was not established experimentally). However, when sound comes from a joint, a common factor involves the movement of joint surfaces with each other because the joints are folded and then instantaneously bloom or the joints are pulled back and repeatedly loosened. ${ }^{(5)} \mathrm{OA}$ is caused by damage to joint cartilage. The destruction of cartilage occurs in the cells that constitute the cartilage, and broken pieces fall off into the joint fluid in the joint to generate friction with the surrounding tissues that leads to inflammation. ${ }^{(6)}$ Cartilage becomes thinner when these actions last for a long time, and the articular cartilage that surrounds the joint surface is eventually worn out and exposes the bones. As a result, the joints are unable to move smoothly. ${ }^{(7,8)}$ Additionally, a joint lesion is a major factor in the destruction of cartilage in an area where pressure is applied. However, new blood vessels grow in the area where pressure is not applied to defend the human body and osteophytes are founded where bones are protruded. ${ }^{(9)}$ The osteophytes limit the movement of the joints and increase pain. ${ }^{(9,10)}$ The exact cause of arthritis is unknown. However, cartilage is damaged by various situations such as obesity or severe exercise that is forcibly performed over a long period of time. ${ }^{(11)}$ The diseases caused by the aforementioned changes are not easy to cure, require longterm treatment, reduce the quality of life, and cause tremendous economic burden. Therefore, the prevention, early diagnosis, and steady treatment of musculoskeletal diseases are necessary to ensure health of elderly individuals. ${ }^{(12)}$

The diagnosis of OA is possible by consolidating several changes in joints with respect to a patient's detailed history and physical and radiological findings. ${ }^{(13)}$ Noninvasive methods, such as X-rays, are the most commonly used radiography techniques that are characterized by decreased joint space due to joint cartilage destruction and loss and osteophyte and subchondral bone sclerosis. ${ }^{(14)}$ Recently, several research studies focused on observing changes in cartilage by directly using MRI and ultrasound to evaluate intra-articular inflammation and intra-osseous changes. ${ }^{(15)}$ Specifically, MRI was used to measure the thickness and volume of cartilage as well as to evaluate the biochemical composition of cartilage, which helps in observing changes in cartilage. However, there are limitations with respect to cost and time, and it is not possible for ultrasound to penetrate bone tissue and to observe a joint as a whole owing to the limitations on the reflection of ultrasonic waves. Additionally, a limit of subjectivity exists owing to a large difference in reproducibility based on the skill of the tester. ${ }^{(16)}$ Invasive methods such as arthroscopy and joint endoscopy use a tube equipped with an ultrasmall camera. ${ }^{(17)}$ Arthroscopy is the X-ray examination of joints that shows the joint structure and morphology. $\mathrm{X}$-ray examination shows an unknown joint surface, a joint cartilage status, and a soft tissue status. However, joints are inevitably considered at various angles because only the plane perpendicular to the radiation is observed. ${ }^{(16)}$ The joint endoscopy method accurately diagnoses parts that cannot be detected by methods such as MRI, CT, and X-rays. However, an endoscope is inserted into the knee joint, and thus, recovery is necessary after diagnosis. Therefore, existing diagnostic methods for arthritis are available in hospitals equipped with expensive equipment but do not conduct measurements during dynamic movements. ${ }^{(18)}$ 
Teague et al. ${ }^{(19)}$ proposed the propagation of an acoustic emission (AE) signal generated inside the body to the skin through fluid-filled internal tissues; a small portion of the acoustic energy is transferred to the atmosphere owing to a high acoustic impedance mismatch between fluid and air. Conversely, most energy is reflected to the tissue, and thus, a piezoelectric filmbased contact microphone attached to the skin surface can be used to measure bone tone. Toreyin et al. ${ }^{(20)}$ used a MEMS-based air microphone that performed measurements $5 \mathrm{~cm}$ away from the surface of the skin because of the signal loss due to motion artifacts when a sensor is attached to the body. Air microphones do not involve signal losses due to motion artifacts, although they are vulnerable to ambient noise. Baczkowicz et al. ${ }^{(21)}$ compared AE signals of normal and chondromalacia patients in the patella using a piezoelectric accelerometer. The variability, amplitude, and significant difference in the chondrogenic grade of AE signals in patients with chondromalacia exceeded those of the normal group. Radzicki and Matsuura ${ }^{(22)}$ confirmed the frequency of the $100 \mathrm{~Hz}-4 \mathrm{kHz}$ region by measuring bone tone with a contact microphone sensor in patients with meniscal tears.

This study involves the diagnosis and monitoring of $\mathrm{OA}$ in the knee in a noninvasive manner by acquiring $\mathrm{AE}$ signals from the knee during dynamic movements using a piezoelectric sensor.

\section{Methods}

\subsection{Bone joint acoustic sensor (BJAS)}

The BJAS was a self-manufactured sensor in Japan. The sensor fixed a pin-type probe (Pogo pin, $\phi 1.5$, and $\ell 5 \mathrm{~mm}$ ) at the center of a unimorph device (Murata Manufacturing Co., Ltd, Japan) in which a disk-shaped piezoelectric ceramic $(\phi 14$ and $t=0.42 \mathrm{~mm})$ and a metal plate $(\phi 20$ and $t=0.42 \mathrm{~mm}$ ) were bonded. A polychloroprene sponge was used as a damper to absorb the vibration energy generated by the unimorph device. In order to attach the sensor to the skin surface, a case ( $\phi 26$ and $h=9.2 \mathrm{~mm}$ ) composed of a 1-mm-thick aluminum alloy fixed with three screws (M1.6 $\times \ell 14 \mathrm{~mm}$ ) was used (Fig. 1). ${ }^{(23)}$

As the knee was moved, vibrations generated in the knee joint were transmitted to the piezoelectric sensor through the probe. This led to the effective mass acceleration of the piezoelectric sensor element, and thus an AE signal was acquired through the principle that charges are generated in proportion to the acceleration.

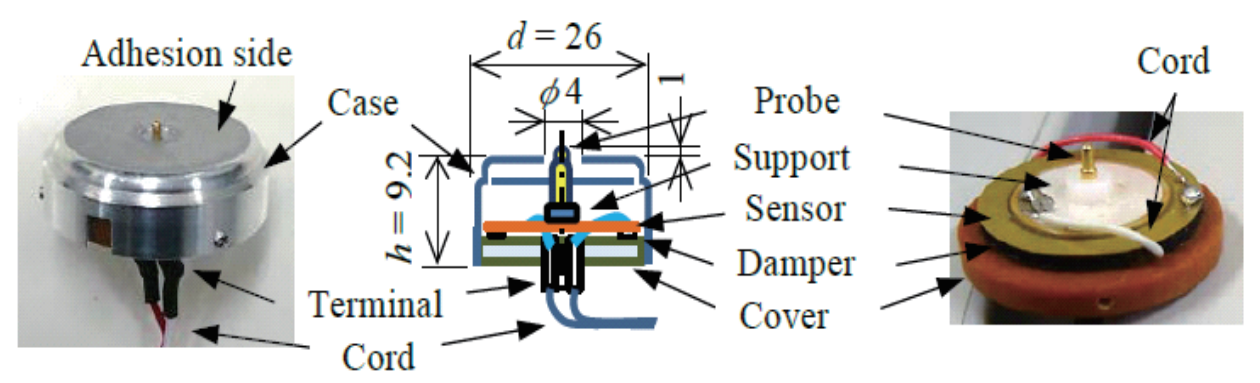

Fig. 1. (Color online) BJAS structure and shape. 


\subsection{BJAS correlation}

In this study, a piezoelectric sensor verification system was constructed to measure the time delay and sensitivity characteristics of output signals between sensors. A sound insulating material $\left(8 \mathrm{~mm}\right.$ thick) was attached to a wooden plate $\left(600 \times 800 \times 50 \mathrm{~mm}^{3}\right)$ to prevent the detection of noise and vibrations from the surroundings. An aluminum plate $(350 \times 200 \times 2$ $\mathrm{mm}^{3}$ ) was placed on the top and a speaker was placed at the center. Four piezoelectric sensors were located at the same distance from the speaker. A silicone foam sheet (hardness 30,2T) was attached to the aluminum plate to mimic the skin surface (Fig. 2).

A sweep wave ( $100 \mathrm{~Hz}$ to $25 \mathrm{kHz}, 3 \mathrm{Vrms}$ amplitude) was transferred to the speaker using a function generator, and the signals from the piezoelectric sensors were recorded at a sampling rate of $50 \mathrm{kHz}$ using a data acquisition system (DAQ) to measure the sensitivity characteristics between the sensors. Although four piezoelectric sensors were located at the same distance from the speaker, it was difficult to simultaneously input signals. Therefore, the differences in the timings of signals received at four sensors were estimated, and the correlation of the signals was then analyzed by cross correlation using the following equations:

$$
R_{x y}(\tau)=\frac{1}{T} \int_{0}^{T} x(t) y(t+\tau) d t
$$

where $x(t)$ and $y(t)$ correspond to two signals, $T$ corresponds to the sampling time of the signals, and $\tau$ corresponds to the time-shift sampling of $y(t)$.

Correlation reflects the extent to which the sensor signals match. If there is no time delay between the two signals, then the peak value appears symmetrically on the basis of $\tau=0$. Cross correlation was used to set the time delay to zero among the four BJASs.

Following this, to evaluate the degree of agreement between the sensors, the correlation between sensors was examined by using an intraclass correlation coefficient (ICC) and a magnitude squared coherence (MSC). The expressions are as follows:

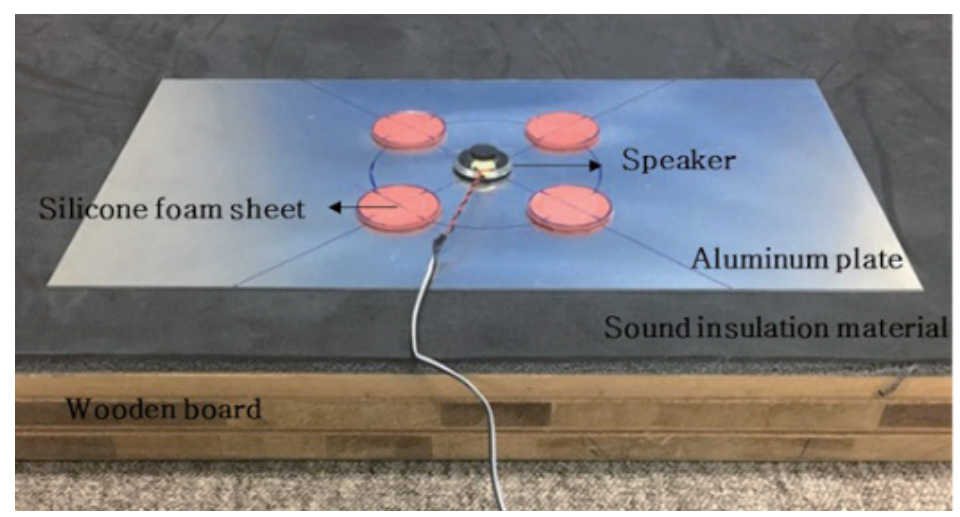

Fig. 2. (Color online) Experimental environment for sensor verification. 


$$
\begin{gathered}
\bar{x}=\frac{1}{2 N} \sum_{n=1}^{N}\left(x_{n, 1}+x_{n, 2}\right), \\
s^{2}=\frac{1}{2 N}\left\{\sum_{n=1}^{N}\left(x_{n, 1}-\bar{x}\right)^{2}+\sum_{n=1}^{N}\left(x_{n, 2}-\bar{x}\right)^{2}\right\}, \\
r=\frac{1}{N s^{2}} \sum_{n=1}^{N}\left(x_{n, 1}-\bar{x}\right)\left(x_{n, 2}-\bar{x}\right),
\end{gathered}
$$

where $x$ corresponds to the average of $N$ paired data values $\left(x_{n, 1, n, 2}\right), s^{2}$ corresponds to the square of standard deviation of $N$ paired data values $\left(x_{n, 1}, x_{n, 2}\right)$, and $r$ corresponds to ICC values of $\left(x_{n, 1}, x_{n, 2}\right)$.

A study by Cicchetti ${ }^{(24)}$ indicated that an ICC above 0.75 denoted an excellent value, an ICC in the range $0.4-0.75$ denoted a good value, and an ICC below 0.4 denoted a moderate value. The equation is as follows:

$$
M S C(f)=\frac{\left|P_{x y(f)}\right|^{2}}{P_{x x(f)} P_{y y(f)}},
$$

where $P_{x y}$ denotes the cross-spectral density between $x$ and $y$, and $P_{x x(f)}$ and $P_{y y(f)}$ denote the autospectral densities of $x$ and $y$, respectively.

If the value of MSC was close to 1, the consistency exists. In this study, sensors 1, 2, and 3 present not only a high ICC correlation coefficient, but also a high MSC value (Table 1, Fig. 3).

\subsection{Participants}

Twenty healthy subjects (11 males and 9 females, age: $25.4 \pm 1.9$ years; body mass: 64.0 $\pm 10.0 \mathrm{~kg}$; height: $169.5 \pm 6.0 \mathrm{~cm}$ ) with no previous injuries or symptoms of swelling or pain and fourteen OA patients diagnosed with Kellgren Lawrence grades I and II (3 males and 11 females, age: $63.0 \pm 8.9$ years; body mass: $63.3 \pm 9.7 \mathrm{~kg}$; height: $162.4 \pm 8.4 \mathrm{~cm})$ participated in the study. Kellgren-Lawrence grading ${ }^{(25)}$ is a representative measure of knee OA. The characteristics of each grade are as follows:

Table 1

ICC values between sensors.

\begin{tabular}{lcccc}
\hline & Sensor 1 & Sensor 2 & Sensor 3 & Sensor 4 \\
\hline Sensor 1 & - & 0.9499 & 0.9410 & 0.7108 \\
Sensor 2 & & - & 0.9950 & 0.6898 \\
Sensor 3 & & & - & 0.6455 \\
Sensor 4 & & & & - \\
\hline
\end{tabular}



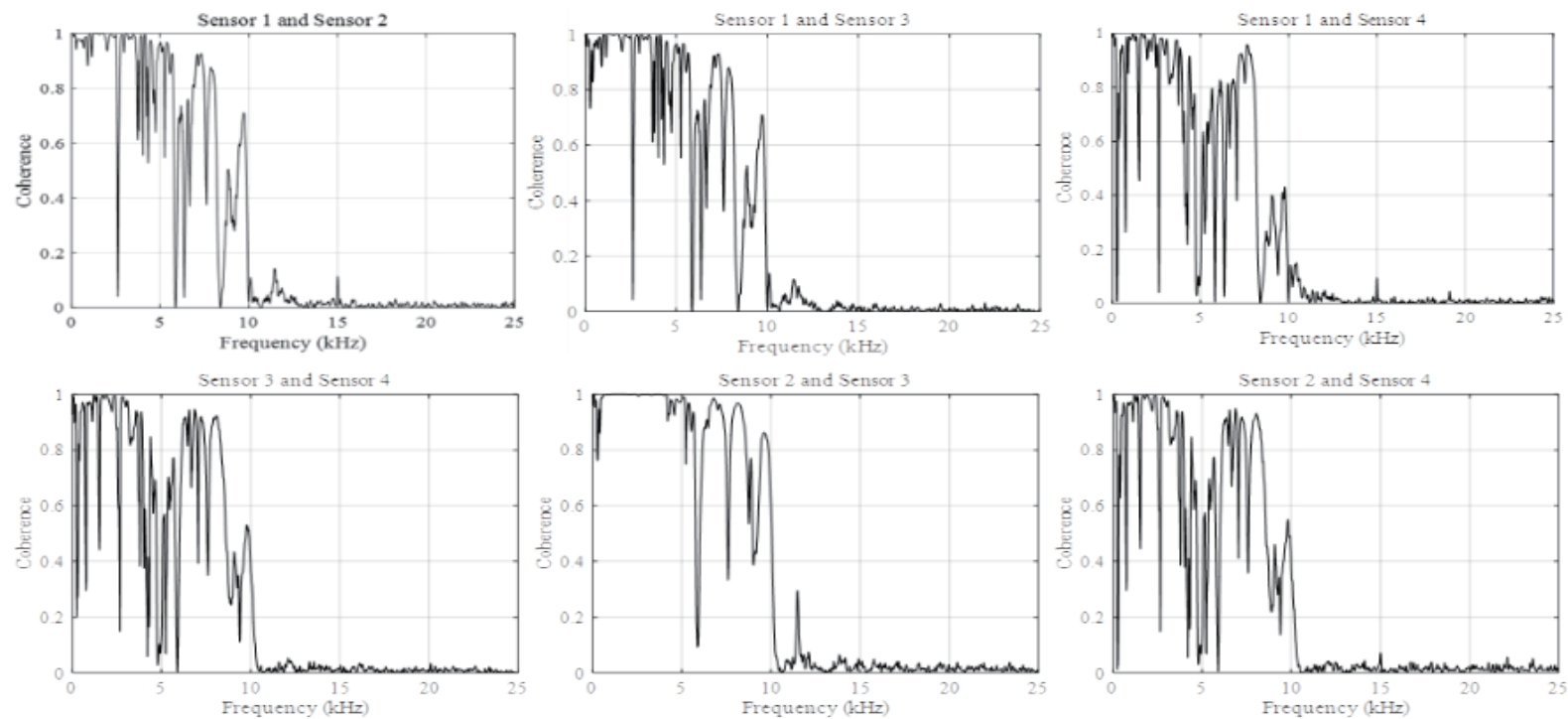

Fig. 3. MSC graphs between two sensors.

grade I: doubtful narrowing of joint space and possible osteophytic lipping;

grade II: definite osteophytes and possible narrowing of joint space;

grade III: moderate multiple osteophytes, definite narrowing of joints space, some sclerosis, and possible deformity of bone contour;

grade IV: large osteophytes, marked narrowing of joint space, severe sclerosis, and definite deformity of bone contour.

Prior to participating in the study, all subjects were informed of the purpose and experimental protocol, and they provided informed and written consent as approved by the Yonsei University Research Ethics Committee (1041849-201704-BM-022-01).

\subsection{Design of testing protocol}

Subjects performed knee flexion/extension exercises on a chair. Three BJASs were attached to the medial epicondyle of the tibia (TM), the lateral epicondyle of the tibia (TL), and the front of the patella (PF) to analyze AE signals from the knee during the experiment. The specific anatomical site exhibits good measurement sensitivity because it is closest to the contact area of the bone surfaces that move together in the knee joint and provides a relatively stable sensor position that is less susceptible to skin motion. Two inertia sensor units (MPU 9150 , Invensens ${ }^{\circledR}$, USA) were attached to the thigh and tibia, and the knee joint angles were calculated using relative angles during the tasks. Three BJASs and two inertia sensor units were simultaneously used to record AEs and knee joint angles at sampling rates of $50 \mathrm{kHz}$ and $100 \mathrm{~Hz}$, respectively (Fig. 4).

During the test, subjects were asked to cross their arms across their chest to eliminate the influence of the movement strategy. Another strategy involved limiting the knee joint angle to 


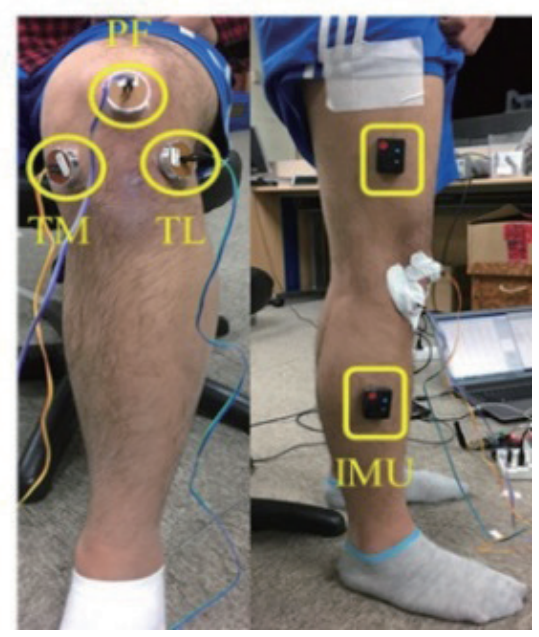

Fig. 4. (Color online) Attachment of BJAS and IMU sensors.

$90^{\circ}$, which is determined by the height of a chair without a backrest and armrests. Exercises were performed to maintain the speed of motion between subjects at a constant rate using a metronome at $30 \mathrm{bpm}$ (a metronome was used to ensure the same speed of motion during the exercise). Each subject's body was stabilized on the chair in the seated position with a knee flexion angle of $90^{\circ}$. Two different tasks were given to each subject: non-weight-bearing (extension-flexion, EF) and weight-bearing (stand-to-sit, STS) exercises. For the non-weightbearing exercise, the subject was asked to extend the knee fully and return to flex the knee with a $2 \mathrm{~s}$ break. Then, for the weight-bearing exercise, the subject stood upright and sat back on a chair. Both tasks provided the range of knee motion of $90^{\circ}$. Both tasks were repeated three times with a 2 min break.

\subsection{Data analysis}

The threshold value of the AE signal was set on the basis of the AE signal recorded for $1 \mathrm{~s}$ in which the subject did not move after the attachment of the piezoelectric sensor (Fig. 5). The magnitude threshold to trigger the $\mathrm{AE}$ recording was set as $m \pm 5 s d$ such that the acquisition system was sufficiently sensitive to collect low-magnitude AE signals observed at the initiation of joint movement. AE signals were converted into sound pressure levels (APLs) in decibels (dB) because of difficulty in observing the differences on the voltage scale. ${ }^{(26)}$ The $\mathrm{dB}$ conversion was made as follows:

$$
A P L=20 \times \log \log _{10} \frac{V}{V_{0}},
$$

where $V$ is the AE signal measured in voltage and $V_{0}$ is the reference voltage considered as the average value of the AE signals recorded for $1 \mathrm{~s}$ while the subject was sitting on the chair. 

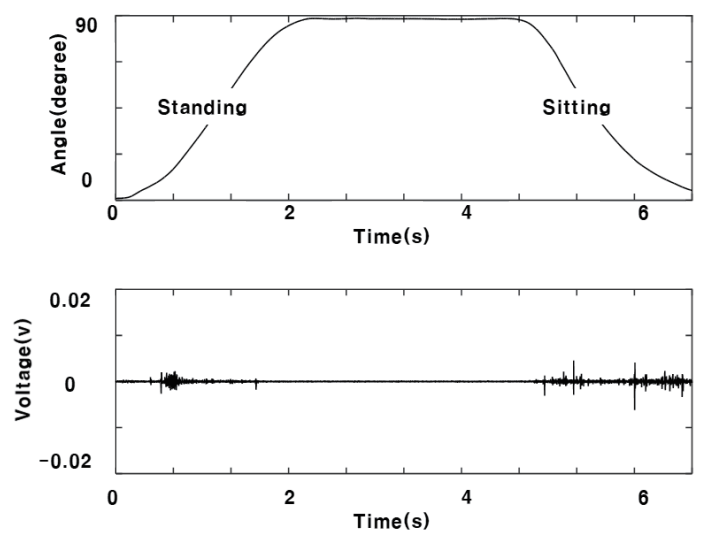

(a)

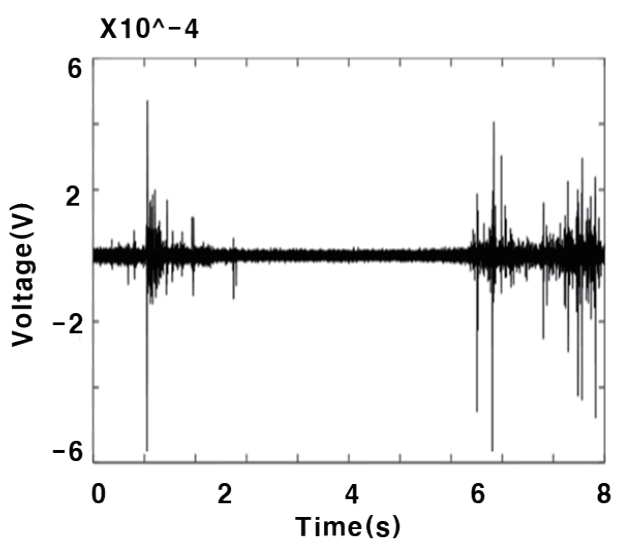

(b)

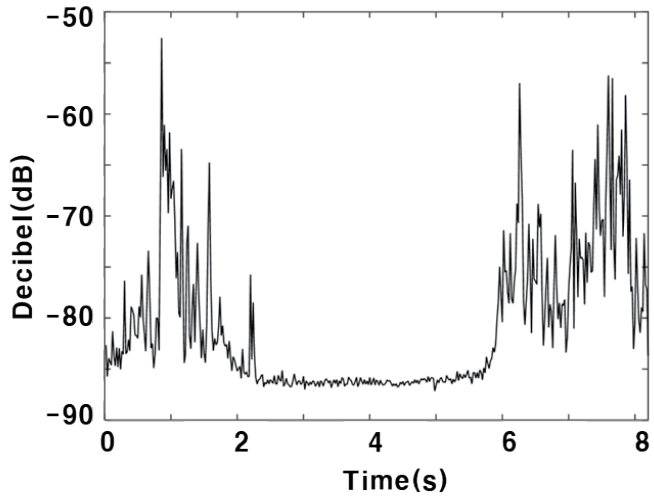

(c)

Fig. 5. (a) Knee flexion angle (top) and raw signal from AE sensor (bottom) during stand-to-sit exercise, (b) raw $\mathrm{AE}$ signals before $\mathrm{dB}$ conversion, and (c) raw AE signals after $\mathrm{dB}$ conversion.

The spectral analysis of the AE signal was performed using short-time Fourier transform (STFT), in which the signal was divided into small sequential or overlapping data frames, and fast Fourier transform (FFT) was applied to each data frame. The output of a continuous STFT provided a time-frequency representation of the signal. In this study, STFT was computed from lengths of 1024 frames using a hamming window. The number of FFT points corresponded to 4069 , and the frames overlapped by $50 \%$.

\subsection{Statistical analysis}

The repeated measure ANOVA (RM-ANOVA) was used to compare differences in the AE signals of two groups. The means and standard deviations were computed for all subjects. For within-group comparisons, the AE signals in a group were compared by using a $t$-test. Statistical analyses were performed with IBM SPSS Statistics (Version 24, IBM, USA), and the significance level was set at $p<0.05$. 


\section{Results}

\subsection{Amplitude and AE event count of signals}

Table 2 shows the frequencies of AE events in the knee joint during STS and EF in each group. The results did not indicate significant differences between STS and EF in individuals in the healthy group. The results indicated significant differences between STS and EF in the OA patients with K-L I and K-L II grades $(p<0.05)$.

The AE events in OA patients exceeded those in the healthy group, and the number of AE events among the groups was statistically significant $(p<0.05)$. However, statistically significant differences between grades I and II were not observed in the OA patients.

Table 3 shows the dB value of AE events in the knee joint during STS and EF in each group. The results did not indicate significant differences between STS and EF in the healthy group. A significant difference between STS and EF was indicated in the K-L I and K-L II grades $(p<0.05)$. When compared with that in the normal group, the AE signal in the OA group exhibited a high $\mathrm{dB}$ value and was statistically significant. However, the results did not indicate statistically significant differences between grades I and II in the OA patients.

\subsection{Frequency characteristics of AE signals}

Figure 6 shows the STFT frequency analysis of the AE signals obtained from the knee joints of the healthy and OA groups during weight-bearing and non-weight-bearing exercises. In the healthy group, the signal was emitted in a frequency range less than $1 \mathrm{kHz}$ and the $\mathrm{dB}$ value was low.

Table 2

$\mathrm{AE}$ events in the knee joint during STS and EF. unit: times

\begin{tabular}{lcccc}
\hline & & TM & TL & PF \\
\hline \multirow{2}{*}{ Healthy group } & STS & $224 \pm 109$ & $395 \pm 261$ & $228 \pm 122$ \\
& EF & $205 \pm 129$ & $240 \pm 102$ & $260 \pm 208$ \\
\hline \multirow{2}{*}{ Grade I } & STS & $1261 \pm 1058$ & $1858 \pm 1132$ & $3301 \pm 1408$ \\
& EF & $341 \pm 268$ & $717 \pm 624$ & $2052 \pm 992$ \\
\hline \multirow{2}{*}{ Grade II } & STS & $1827 \pm 788$ & $1015 \pm 994$ & $3765 \pm 1195$ \\
& EF & $731 \pm 452$ & $421 \pm 439$ & $2402 \pm 921$ \\
\hline
\end{tabular}

Table 3

$\mathrm{dB}$ values of AE signals in the knee joint during STS and EF. unit: $\mathrm{dB}$

\begin{tabular}{lcccc}
\hline & & TM & TL & PF \\
\hline \multirow{2}{*}{ Healthy group } & STS & $-85.5 \pm 0.3$ & $-85.2 \pm 0.6$ & $-85.6 \pm 0.3$ \\
& EF & $-85.3 \pm 0.4$ & $-85.3 \pm 0.4$ & $-85.5 \pm 0.5$ \\
\hline \multirow{2}{*}{ Grade I } & STS & $-82.8 \pm 2.3$ & $-82.0 \pm 2.2$ & $-77.2 \pm 4.0$ \\
& EF & $-84.9 \pm 0.9$ & $-84.0 \pm 2.0$ & $-79.4 \pm 4.3$ \\
\hline \multirow{2}{*}{ Grade II } & STS & $-82.6 \pm 1.5$ & $-83.9 \pm 2.2$ & $-78.3 \pm 3.3$ \\
& EF & $-83.9 \pm 1.1$ & $-84.9 \pm 0.9$ & $-77.9 \pm 4.1$ \\
\hline
\end{tabular}




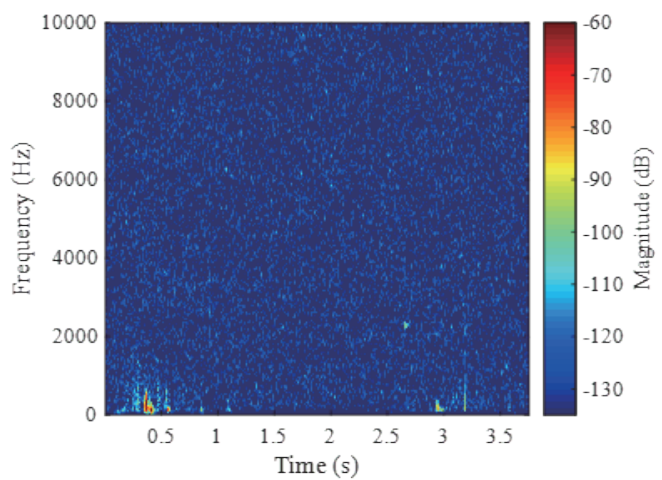

(a)

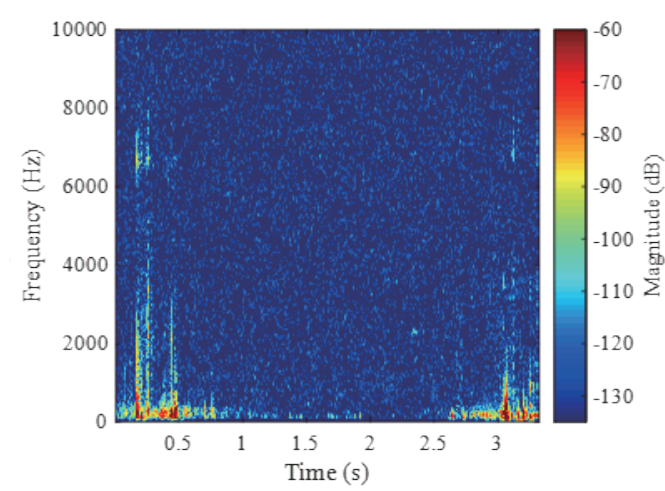

(c)

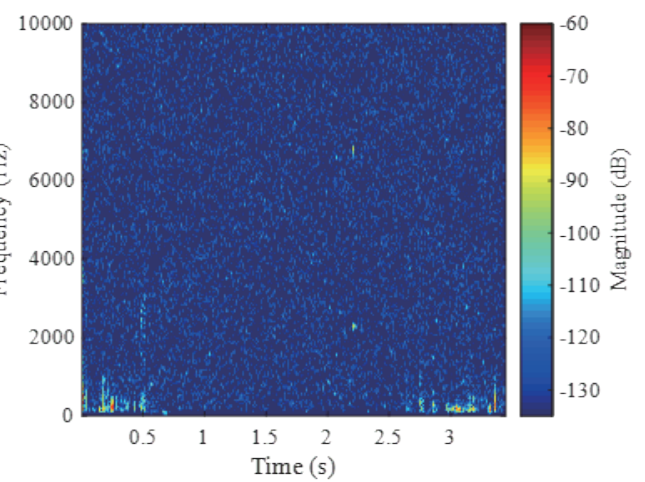

(b)

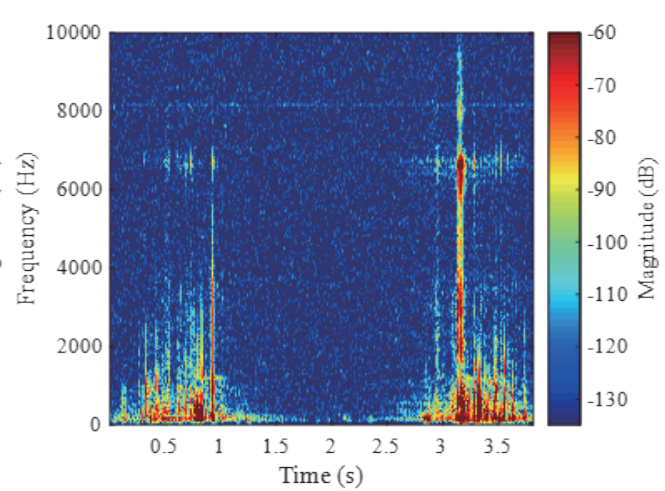

(d)

Fig. 6. (Color online) (a) Normal group of STFT during non-weight-bearing exercises, (b) normal group of STFT during weight-bearing exercises, (c) patient group of STFT during non-weight-bearing exercises, and (d) patient group of STFT during weight-bearing exercises.

In the $\mathrm{OA}$ group, the $\mathrm{AE}$ signal was detected in a frequency range below $10 \mathrm{kHz}$, and the $\mathrm{dB}$ value exceeded that in the healthy group. Additionally, it was observed that the $\mathrm{dB}$ value detected in the weight-bearing exercise was higher than that in the non-weight-bearing exercise.

\section{Discussion}

In this study, the characteristics of AE signals were analyzed in OA patients and healthy adults. Toreyin et al. ${ }^{(20)}$ suggested that healthy individuals could use a microphone to analyze the AE signal in the knee by removing background noise in uncontrolled surroundings. However, the results of the current study indicated that it was not necessary to eliminate artificial background noise when using a piezoelectric sensor that was not affected by ambient noise.

Smith ${ }^{(27)}$ reported that the bending velocity of the hip joint varied with the standing speed of a chair and thus potentially affected the leg strength of the legs. Hwang et al. ${ }^{(28)}$ also showed that the height of a chair affected lower extremity muscles and knee joints during the standing motion. Therefore, in this study, to eliminate the influence of speed, the movement is limited 
to the sound of the metronome that is adjusted to $30 \mathrm{bpm}$. To reduce the influence on the height of the chair, the height of the chair was set such that the knee angle corresponded to $90^{\circ}$ when a subject was seated.

In this study, the AE signals were measured by attaching sensors to the medial epicondyle of the tibia, the lateral epicondyle of the tibia, and the front of the patella in the same position as the previous studies. ${ }^{(19-22)}$ Two IMU sensors were also attached to the thigh and the tibia to calculate knee joint angle because an IMU sensor has the advantages of low price, small size, and light weight, unlike camera-based motion analysis systems.

Baczkowics et al. ${ }^{(21)}$ analyzed AE signals in normal knee and chondromalacia patients during knee flexion and extension. The results indicated that the amplitude of the AE signal was larger in the patients compared to those in the normal group, and frequencies were confirmed in the $100 \mathrm{~Hz}-1 \mathrm{kHz}$ range. The AE event count and the $\mathrm{dB}$ values in the $\mathrm{OA}$ patients exceeded those in healthy individuals, and this confirmed that the amplitude of the signals in the patient group exceeded those in the previous study. However, in this study, the frequency range of the AE signals was from $100 \mathrm{~Hz}$ to $10 \mathrm{kHz}$.

Teague et al. ${ }^{(19)}$ attached four AE sensors to the knees of patients with anterior cruciate ligament rupture. The optimal location through the extension of the knee and flexion movement was termed the medial knee. However, in this study, the AE signal analysis of OA patients revealed that $\mathrm{AE}$ signals differed with the lesion site. In contrast, in most patients, the $\mathrm{AE}$ signal significantly increased in the vicinity of PF. It was not possible to determine the optimal position to obtain the correct AE signal according to the patient.

In this study, a small number of subjects and the absence of age matching between patient and normal groups pose limitations. Additionally, the AE signals of the subjects were significantly different among individuals and did not exhibit any significant differences between patients with grade I and grade II OA. To increase the reliability of the results, it will be necessary to first quantitatively evaluate the condition of a subject through X-ray photographs in future studies. Furthermore, for the standardization of these results, not only grades I and II, but also grades III and IV should be considered in future studies.

\section{Conclusions}

In this study, knee AE signals were successfully measured using piezoelectric sensors in patients with OA (Kellgren Lawrence grades I and II) and healthy adults. First, the number of $\mathrm{AE}$ events and $\mathrm{dB}$ values in patients with $\mathrm{OA}$ was statistically significantly higher than those in the normal group. Second, the frequency of AE signals was detected in the region below $1 \mathrm{kHz}$ in the normal group and the region corresponding to $100 \mathrm{~Hz}-10 \mathrm{kHz}$ in the OA patients. Third, in the normal group, the $\mathrm{AE}$ events and the $\mathrm{dB}$ values were relatively small, and there was no significant difference between the weight-bearing exercise and the non-weight-bearing exercise. Finally, in the OA group, the AE events resulted in more counts and larger $\mathrm{dB}$ values for $\mathrm{AE}$ signals in the lesion areas. There were no significant differences between grades I and II of OA patients, although it was confirmed that values were higher in the weight-bearing exercise than in the non-weight-bearing exercise. 
It is extremely difficult to determine a disease using piezoelectric sensors because the AE of the knee joint is high with respect to differences in individuals. However, instead of expensive equipment (such as those used in X-rays or arthroscopies), it is expected that sensors can be used to monitor knee conditions simply before or at the beginning of a disease.

\section{Acknowledgments}

This research was supported by The Leading Human Resource Training Program of Regional Neo Industry through the National Research Foundation of Korea (NRF) funded by the Ministry of Science, ICT and Future Planning (No. 2016H1D5A1909760).

\section{References}

1 B. Heidari: Caspian J. Intern. Med. 2 (2011) 205.

2 T. Aigner, J. Rose, J. Martin, and J. Buckwalter: Rejuvenation Res. 7 (2004) 134.

3 A. S. Voloshin and J. Wosk: J. Biomed. Eng. 5 (1983) 157.

4 K. Lund-Olesen: Arthritis Rheumatol. 13 (1970) 769.

5 G. N. Kawchuk, J. Fryer, J. L. Jaremko, H. Zeng, L. Rowe, and R. Thompson: PLoS One 10 (2015) e0119470.

6 J. E. Tarride, M. Haq, D. J. O'reilly, J. M. Bowen, F. Xie, L. Dolovich, and R. Goeree: Arthritis Rheumatol. 64 (2012) 1153.

7 G. Peat, R. Mc-Carney, and P. Croft: Ann. Rheum. Dis. 60 (2001) 91.

8 D. T. Felson: N. Engl. J. Med. 354 (2006) 841.

9 T. Hayami, M. Pickarski, GA. Wesolwski, J. Mclane, A. Bone, J. Destefano, G. A. Rodan, and L. T. Duong: Arthritis Rheumatol. 50 (2004) 1193.

10 S. C. O’Reilly, K. R. Muir, and M. Doherty: Ann. Rheum. Dis. 55 (1996) 931.

11 P. Creamer, M. Lethbridge-Cejku, and MC. Hochberg: J. Rheumatol. 26 (1999) 1785.

12 H. A. Israel, F. Saed-Nejad, and A. Ratcliffe: J. Oral Maxillofac. Surg. 49 (1991) 708.

13 K. D. Brandt, R. S. Fife, E. M. Braunstein, and B. Katz: Arthritis Rheumatol. 34 (1991) 1381.

14 I. F. Petersson, T. Boegard. T. Saxne, A. J. Silman, and B. Svensson: Ann. Rheum. Dis. 56 (1997) 493.

15 D. K. Hunter, G. H. Lo, D. Gale, A. J. Grainger, and P. G. Conaghan: Ann. Rheum. Dis. 67 (2008) 206.

16 J. Duryea, G. Neumann, J. Niu, S. Totterman, J. Tamez, C. Dabrowski, and D. J. Hunter: Arthritis Care Res. 62 (2010) 932.

17 S. L. Brooks, J. W. Brand, S. J. Gibbs, L. Hollender, A. G. Lurie, K. A. Omnell, and S. C. White: Oral Surg. Oral Med. Oral. 83 (1997) 609.

18 M. A. Roling, M. I. Visser, E. H. Oei, P. Pilot, G. J. Kleinrensink, and R. M. Bloem: BMC Musculoskeletal Disorders 16 (2015) 50.

19 C. Teague, S. Hersek, H. Toreyin, M. L. Millard-Stafford, M. L. Jones, G. F. Kogler, and O. T. Inan: Proc. 2015 IEEE 12th Int. Conf. Wearable and Implantable Body Sensor Networks (IEEE, 2015) 1.

20 H. Toreyin, S. Hersek, C. N. Teague, and O. T. Inan: IEEE Sens. 16 (2016) 2892.

21 D. Baczkowicz, E. Majorczyk, and K. Krecisz: Biomed Res. 2015 (2015) 591707.

22 V. Radzicki and K. Matsuura: Int. Telemetering Conf. Proc. 51 (2015).

23 M. Nagao, S. I. Konno, Y. H. Kim, and O. Yokota: Technol. Health Care 23 (2015) 715.

24 D. V. Cicchetti: Psychol Assessment 6 (1994) 284.

25 J. H. Kellgren and J. S. Lawrence: Ann. Rheum. 16 (1957) 494.

26 G. Shen, Z. Wu, and J. Zhang: Advances in Acoustic Emission Technology: Proc. World Conf. Acoustic Emission (Springer, 2015) 358.

27 M. Smith: Physiotherapy 67 (2015) 215.

28 S. J. Hwang, J. S. Son, J. Y. Kim. H. D. Kim, and Y. H. Kim: J. Biomed. Eng. Res. 29 (2008) 484. 


\section{About the Authors}

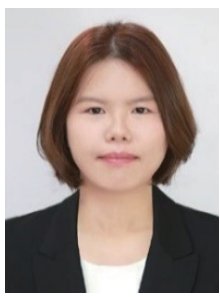

Dagyeong Choi received her B.S. in Biomedical Engineering from Konyang University in 2014. She is currently an M.S. student in the Department of Biomedical Engineering at Yonsei University, Korea. Her research interests are in the areas of rehabilitation engineering, sensors, and bio-signal analysis.

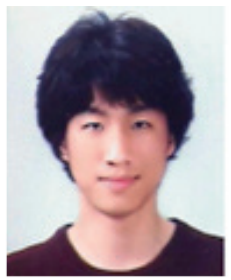

Soonjae Ahn received his B.S. and M.S. degrees in Biomedical Engineering from Yonsei University in 2011 and 2013, respectively. He is currently a Ph.D. student in the Department of Biomedical Engineering at Yonsei University, Korea. His research interests are in the areas of rehabilitation engineering and human movement.

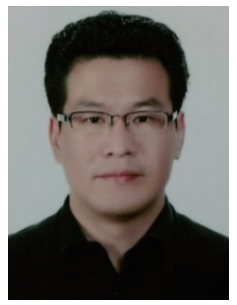

Jeseong Ryu received his B.S. degree from Dept. of Mechanical Engineering, Hanyang University, Korea, in 2001. From 2003 to 2009, he worked as a researcher at the R\&D center in a company. He received his M.S. and Ph.D. degrees from the Department of Biomedical Engineering, Yonsei University, Korea, in 2012 and 2017, respectively. His research interests are biomechanics, rehabilitation engineering, and prosthetics and orthotics.

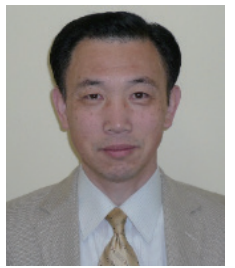

Mitsuo Nagao received his B.S. degree from Nihon University, Japan, in 1979 and his M.S. and Ph.D. degrees from Nihon University, Japan, in 1983 and 2010, respectively. From 2012 to 2015, he was an assistant professor in Nihon University, Japan. Since 2016, he is a professor in Nihon University. His research interests include MEMS, bioengineering, and sensors.

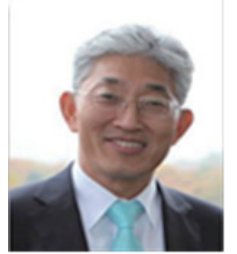

Youngho Kim received his B.S. degree in Mechanical Engineering from Hanyang University in 1982. He received his M.S. and Ph.D. degrees in Biomedical Engineering from the University of Iowa in 1989 and 1991, respectively. He is currently a professor in the Department of Biomedical Engineering at Yonsei University, Korea. His research interests are in the areas of human movement, rehabilitation engineering, and biomechanics. 